\title{
Training in Endoscopy: Enteroscopy
}

\author{
Jinsu Kim \\ Division of Gastroenterology, Department of Internal Medicine, Seoul St. Mary's Hospital, College of Medicine, The Catholic University of \\ Korea, Seoul, Korea
}

\begin{abstract}
The balloon-assisted enteroscope has been regarded as the standard device for direct visualization of deep small bowels and allows for the diagnosis and treatment of small bowel disease. At the beginning, its application was focused on the diagnosis of obscure gastrointestinal bleeding, inflammatory bowel disease, and small bowel tumor. However, the indications are being expanded to various therapeutic procedures, not only confined to bleeding control. With the expansion of the indications, the need to perform enteroscopy effectively and safely is increasing. Recent studies have been focused on the diagnostic yield, therapeutic yield, and longterm outcomes of the device. However, with the increasing number of procedures, procedural guidelines and quality indicators are also needed. Clin Endosc 2017;50:328-333
\end{abstract}

Key Words: Enteroscope; Insertion technique; Single-balloon enteroscopy; Double-balloon enteroscopy

\section{INTRODUCTION}

In general, the duodenum and part of the terminal ileum can be reached by a conventional gastroscope and colonoscope. However, the small bowel, deeper than the duodenum and terminal ileum, has been considered as an inaccessible area for a long time since the development of flexible endoscopes, as the small bowel is approximately four times longer than the colon and loops are inevitably formed during deep endoscopic insertion. ${ }^{1}$ Several methods have been developed to overcome these problems. In March 1972, Hiratsuka et al. ${ }^{2}$ reported the world's first successful total enteroscopy using the ropeway method. Another device that enables total enteroscopy (Sonde method) was also developed several years later. ${ }^{3}$ However, both methods were not widely used because of patient discomfort and because they were labor intensive and

Received: June 2, 2017 Revised: July 22, 2017

Accepted: July 24, 2017

Correspondence: Jinsu Kim

Division of Gastroenterology, Department of Internal medicine, Seoul St. Mary's Hospital, College of Medicine, The Catholic University of Korea, 222 Banpo-daero, Seocho-gu, Seoul 06591, Korea

Tel: +82-2-2258-6070, Fax: +82-2-2258-2083, E-mail: jinsoo@catholic.ac.kr

(cc) This is an Open Access article distributed under the terms of the Creative Commons Attribution Non-Commercial License (http://creativecommons.org/ licenses/by-nc/3.0) which permits unrestricted non-commercial use, distribution, and reproduction in any medium, provided the original work is properly cited. time consuming. After the 21st century, capsule endoscopy and balloon-assisted enteroscopy (BAE) have been developed and widely used for the diagnosis and treatment of small bowel disease, including obscure gastrointestinal bleeding, small bowel tumor, inflammatory bowel disease, and drug-induced enteropathy. ${ }^{4}$ Even though endoscopists have extensive experience with upper endoscopy and colonoscopy, and mastered both procedures, specific techniques are necessary for them to perform enteroscopy. Recently, many reports and review articles have focused on the diagnostic yield, therapeutic yield, and long-term outcomes of the device. However, only few articles have described learning and performing enteroscopy. This paper focuses on the real practice of enteroscopy, especially double-balloon enteroscopy (DBE) and single-balloon enteroscopy (SBE), which is widely used in most countries.

\section{BALLOON-ASSISTED ENTEROSCOPY}

\section{DBE system}

Double- and single-balloon enteroscopies are the most popular methods of BAE. Yamamoto et al. introduced DBE and reported on its use for total small bowel endoscopy that enabled tissue biopsy and endoscopic therapy. ${ }^{5}$ The DBE system is composed of a high-resolution flexible enteroscope with a 
35-mm diameter latex balloon attached to the end of the enteroscope and an additional latex balloon attached to the tip of the overtube made of polyurethane. Both balloons are inflated and deflated using an external pressure-controlled pump system. Currently, several DBE systems for different indications are available. The P type (EN-450P5; Fujinon, Saitama, Japan) is the thinnest, with an outer diameter of $8.5 \mathrm{~mm}$, and its high flexibility enables deep insertion and complete enteroscopy. However, the diameter of its working channel is only $2.2 \mathrm{~mm}$, so it is not suitable for performing therapeutic procedures such as hemostasis, polypectomy, and endoscopic clipping. The T type (EN-450T5, EN-580T; Fujinon, Saitama, Japan) has a larger outer diameter $(9.4 \mathrm{~mm})$ and larger working channel diameter (2.8 or $3.2 \mathrm{~mm}$ ). The short type (EC450-B15; Fujinon, Saitama, Japan) with a length of $152 \mathrm{~cm}$ is useful for endoscopic retrograde cholangiopancreatography (ERCP) for an altered anatomy, and difficult or failed colonoscopy. The main advantage of the short type is that it does not require specially designed accessories for performing an enteroscopic procedure. Almost all conventional devices are available in the short-type DBE.

\section{SBE system}

SBE was introduced in 2007 as an alternative to DBE. SBE is a simpler device than DBE because the balloon at the tip of the enteroscope is not present. As such, SBE is regarded as a more convenient tool than DBE, as only a single balloon is attached to the overtube; therefore, the process of manipulation of the balloon control unit is simplified. The SBE system consists of the SIF-Q180 enteroscope (Olympus Optical, Tokyo, Japan), overtube balloon control unit (Olympus Balloon Control Unit) and a silicone overtube and balloon (ST-SB1). The enteroscope is a high-resolution video endoscope that works with Olympus EVIS processors and the EVIS EXERA II system. The outer diameter is $9.2 \mathrm{~mm}$, with a working length of $2,000 \mathrm{~mm}$ and working channel size of $2.8 \mathrm{~mm}$. The overtube and balloon made of silicon material can be helpful in patients with latex allergy, which could occur during the use of the DBE system. The flushing of the overtube with a small amount of water activates the hydrophilic coating and thereby prevents friction between the overtube and the enteroscope. The balloon is inflated and deflated by a balloon control unit with a safety pressure setting that ranges from -6.0 to +5.4 $\mathrm{kPa}$.

\section{INSERTION TECHNIQUE}

\section{General principle}

Both DBE and SBE use the push-and-pull method. Howev- er, the number of balloon devices differ, which can make a little difference in the insertion technique between the two BAE systems. DBE is generally performed by two persons. The operator controls the enteroscope, and the assistant manipulates the overtube. For the antegrade approach (oral route), the endoscope and overtube are advanced to the duodenum, past the major papilla, and the overtube balloon is inflated to hold the small bowel tightly. The enteroscope is then advanced to the distal side of the small bowel, and its balloon is inflated to hold the stable position and prevent slippage of the scope backward. The overtube balloon is then deflated, and the overtube is advanced toward the tip of the enteroscope. The overtube balloon is then reinflated. With this process, the two balloons could hold the intestine more tightly. The enteroscope-overtube is then withdrawn to fold the small intestine along the overtube. This process is then repeated until maximal insertion point or the target site is reached. Most of the small bowel has no distinguishing features. A marking of such points by India ink tattooing or clipping may be often needed.

SBE is also usually performed by two persons. The enteroscope and overtube are advanced to the proximal jejunum similar to the DBE insertion technique. However, no enteroscope balloon enables holding the position during manipulation of the overtube. An angulation of the enteroscope tip during advancement of the overtube is needed. After angulation of the tip, the balloon of the overtube is deflated, and the overtube is advanced toward the tip of the enteroscope. The overtube balloon is then reinflated. The enteroscope-overtube is then withdrawn to fold the small intestine along the overtube. The overtube balloon is left inflated to tightly hold the position and deliver pushing forces to the tip of the enteroscope without looping. The enteroscope is then advanced approximately $40 \mathrm{~cm}$ from the overtube tip. This push-pull cycle of forward advancement and withdrawal is repeated until the maximal insertion point or target lesion is reached. ${ }^{6}$

\section{Detailed insertion technique}

\section{Retrograde insertion technique}

Retrograde insertion is more difficult than antegrade insertion, even for expert endoscopists. In case of DBE, the enteroscope and overtube are advanced to the cecum with the push-and-pull technique. After inflation of the balloon, the enteroscope-overtube is withdrawn to decrease the sharp ileocolic angle. With the overtube balloon inflated, the enteroscope is then passed through the ileocecal valve, with its balloon inflated within the ileum to hold the position. The overtube is then advanced into the ileum with the balloon deflated. In case of SBE, an insertion in the terminal ileum can be performed similarly to that in DBE. However, backward 


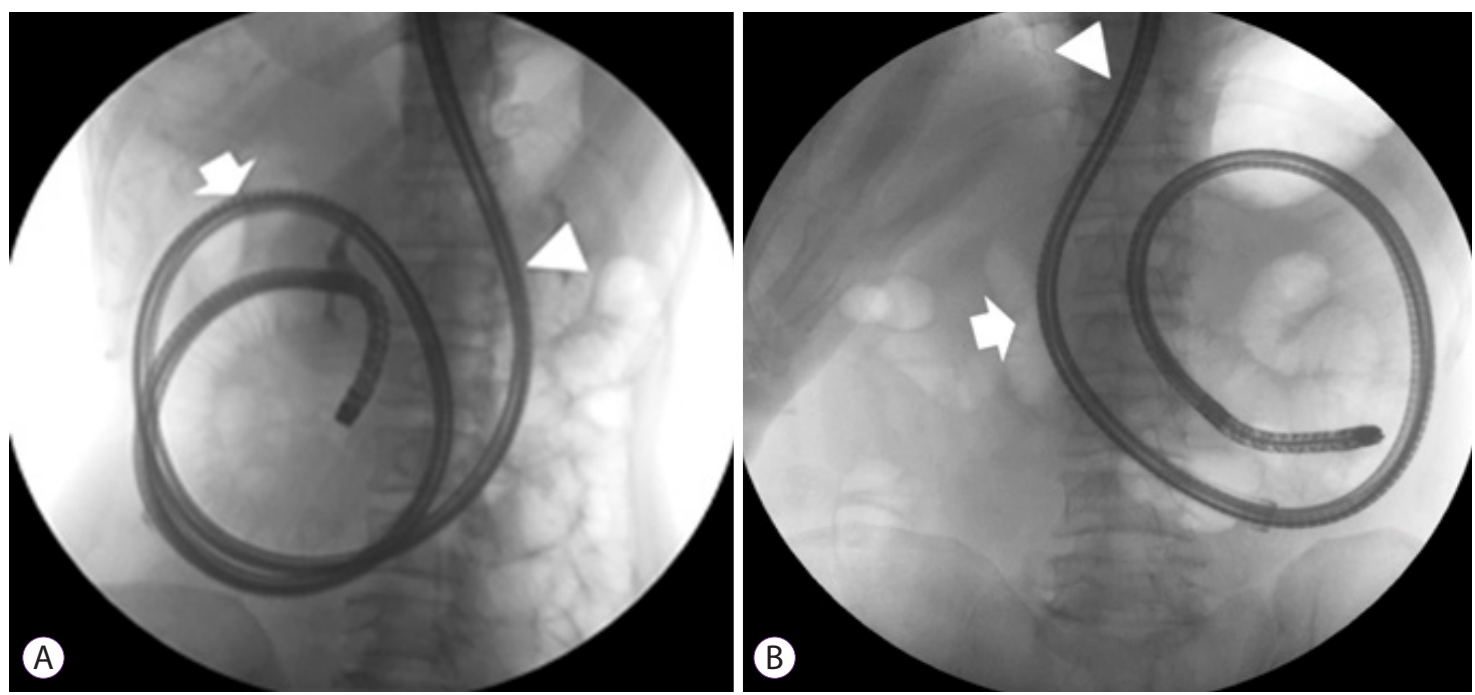

Fig. 1. Clockwise or counterclockwise rotation. (A) In clockwise rotation, a large intragastric loop recurred when the scope was pushed to advance it (arrow: pyloric ring, arrowhead: esophagogastric junction). (B) In the counterclockwise rotation, the intragastric loop was effectively controlled without recurrence during insertion.

slippage of the tip of the enteroscope to the cecum during insertion of the overtube may frequently occur because of lack of an enteroscope balloon, which enables holding the position. To overcome this problem, enough insertion of the enteroscope tip in the terminal ileum is necessary. Sometimes, the scope cannot be successfully inserted in the terminal ileum. Abdominal compression and position change may be helpful in such a case.

\section{Counterclockwise insertion}

For deep insertion, the enteroscope makes a circular small bowel loop. It could be counterclockwise or clockwise. The direction of the enteroscope rotation could be a combination of counterclockwise and clockwise rotations during insertion of the scope in the proximal small bowel. After the shortening procedure, the enteroscope could be rotated clockwise or counterclockwise. We could insert the scope regardless of direction, but in case of a clockwise rotation, a large intragastric loop usually recurred when we pushed the scope to advance it more, hindering an effective deep insertion of the scope without unwanted looping. In cases of counterclockwise rotation, an intragastric looping usually did not occur when we pushed the scope to advance and enable effective deep insertion of the scope (Fig. 1). Another potential disadvantage of the clockwise rotation is post-BAE acute pancreatitis, which could occur as a result of the severe mechanical strain from traumatic injury and/or ischemia on the pancreatic body and tail.

\section{Single-man method}

BAE is generally performed by two persons who must cooperate closely. Although the procedure by two persons has been regarded as the standard, considering the transition

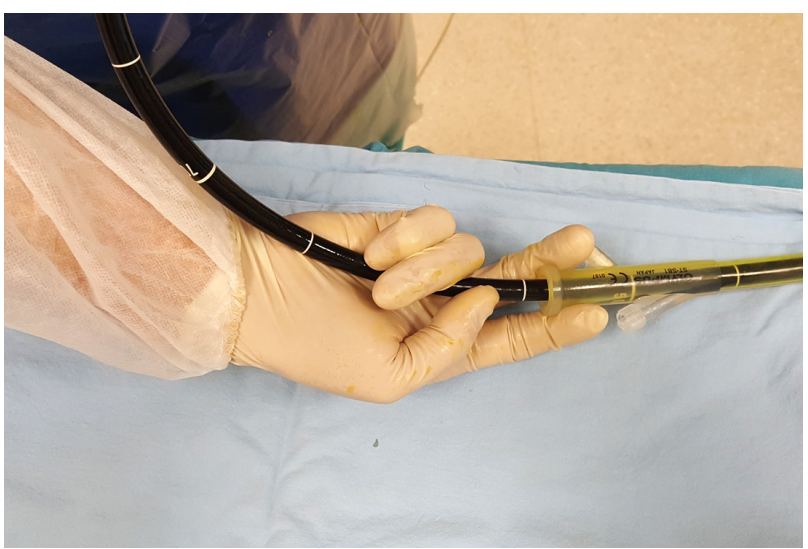

Fig. 2. Grip and pinch technique: The operator grips the enteroscope behind the overtube, between the third and fifth fingers, and the palm and pinches the overtube by using the first and second fingers.

from the two- to the one-person method in colonoscopy, the single-man method may be available in enteroscopy procedures. Araki et al. ${ }^{8}$ described the "grip and pinch" technique. The operator "grips" the enteroscope behind the overtube between the third to fifth fingers and palm, and "pinches" the overtube by using the first and second fingers (Fig. 2). SBE is technically easier to perform because the operator controls only one balloon and a simplified balloon control unit, and may be more appropriate for the single-man method.

\section{Decision on the optimal insertion direction after contrast infusion}

To transfer the pushing force to the distal end of the enteroscope, avoiding a sharp angulation during insertion may be necessary. Frequently, sharp angulation of the small bowel loop prevents further advancement of the enteroscope and only stretches the small bowel. When we visualize the scope 

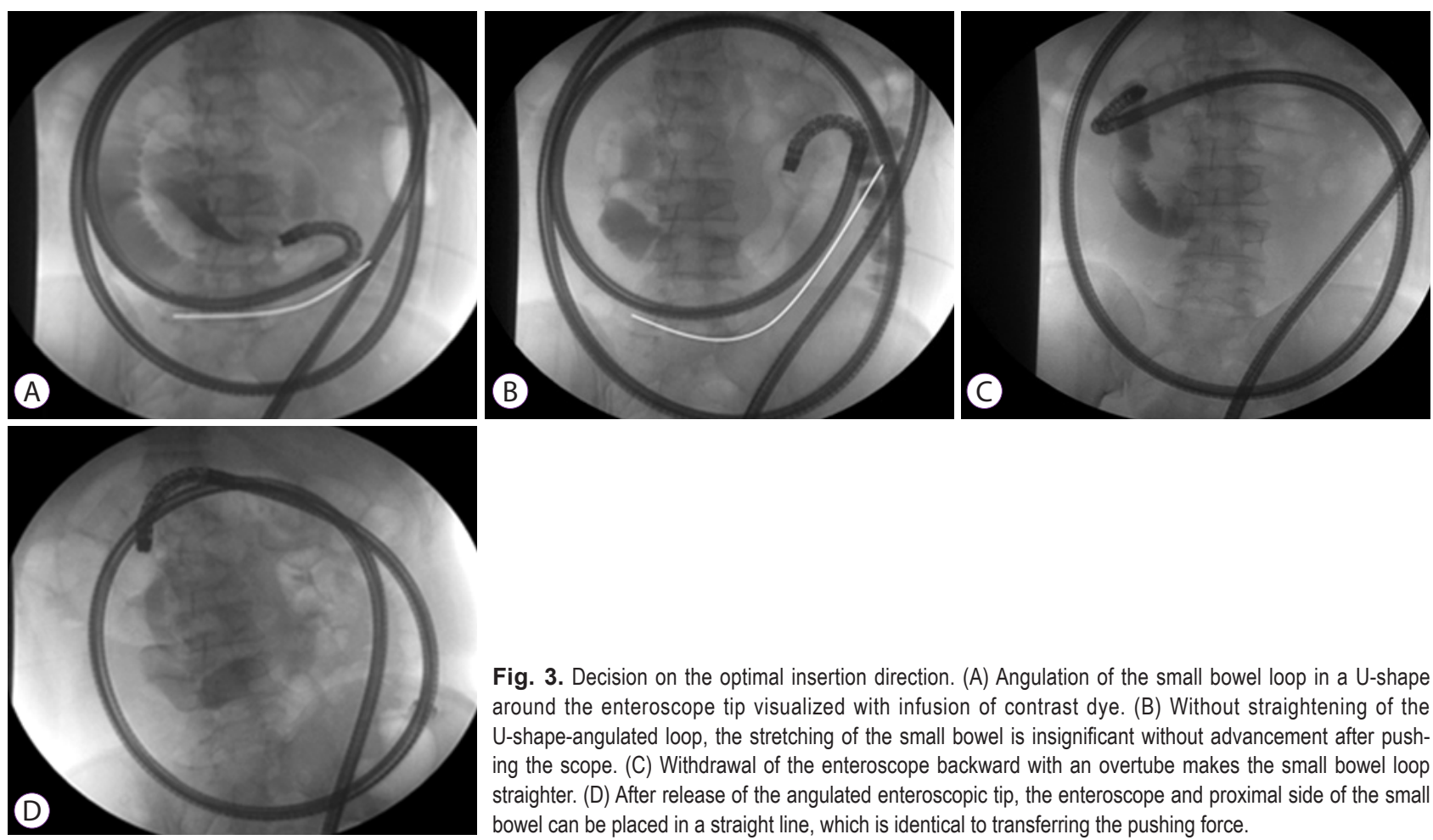

Fig. 3. Decision on the optimal insertion direction. (A) Angulation of the small bowel loop in a U-shape around the enteroscope tip visualized with infusion of contrast dye. (B) Without straightening of the U-shape-angulated loop, the stretching of the small bowel is insignificant without advancement after pushing the scope. (C) Withdrawal of the enteroscope backward with an overtube makes the small bowel loop straighter. (D) After release of the angulated enteroscopic tip, the enteroscope and proximal side of the small bowel can be placed in a straight line, which is identical to transferring the pushing force.

with a fluoroscope, a more counterclockwise rotation of the scope seems to attain greater advancement of the scope. However, for angulation, pushing force could not be effectively transferred to the distal side of the scope, resulting in only meaningless stretching of the intestine. By contrast dye infusion, we could predict the insertion direction. When we encounter sharp angulation, withdrawal of the enteroscope backward with an overtube makes the small bowel loop straighter. Through visualization of the more proximal side of the small bowel with a contrast dye, we can monitor how the proximal side is straightened and decide when to stop the shortening procedure and repush the scope (Fig. 3).

\section{Fluoroscopy guidance}

Fluoroscopy guidance may be helpful to verify the location and direction of the rotation of the scope. In the author's opinion, fluoroscopy guidance is necessary until physicians get used to the procedure. Once the physicians obtain extensive experience, it can be performed without fluoroscopy guidance. However, in case of severe adhesion, fluoroscopy assistance may be needed to check the proper direction of the scope, although the operators are expert in BAE.

\section{Power suction method}

To prevent backward slippage of the scope during insertion of the overtube, the power suction method to hold the scope position may be helpful. ${ }^{9}$ However, it could make mucosal suction injury similar to red spots or angiodysplasia. If the indication of enteroscopy was for searching for a recurrent bleeding lesion, which was not obvious in a previous capsule endoscopy, such mucosal injury by suctioning may be confused with tiny angiodysplasia. Therefore, in the author's opinion, in such indication, we should reconsider the use of the power suction method.

\section{Simultaneous technique}

This technique consists of withdrawing the inflated overtube to fold the intestine and simultaneously pushing the scope into the deep small bowel. This method showed a shorter mean procedural time, but the depth of insertion was not improved by this method. However, this technique could be helpful in passing the scope through the adhesion point by reducing the loop formation during scope insertion. ${ }^{10,11}$

\section{CONSIDERATIONS OTHER THAN INSERTION}

\section{Overtube injury}

During withdrawal of the enteroscope, we could sometimes find a mucosal injury or laceration not found during scope insertion. It is usually due to injury by the overtube, and the mechanism is similar to injury by the colonoscope shaft, which could leave a mucosal laceration or sometimes even 


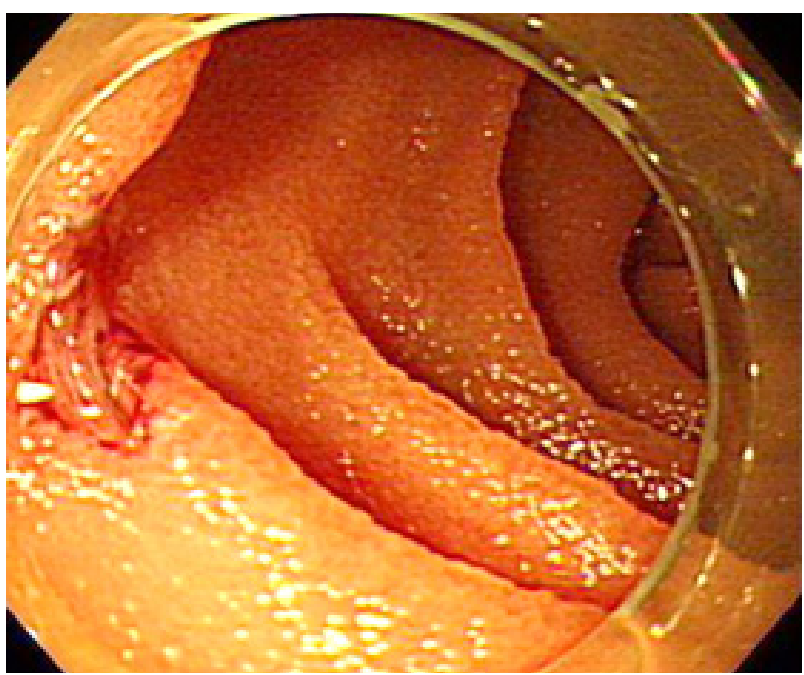

Fig. 4. Exposure of the muscle layer due to mucosal injury by the overtube.

perforation by excessive loop formation during insertion. Mucosal injuries are usually found proximal to the small bowel and could be left without specific management if no signs of perforation are found. In the author's opinion, the most common site is the area around the ligament of Treitz, which shows sharp angulation during scope insertion. Repeated friction during the push-and-pull procedure of the overtube and stretching of the intestine due to loop formation during the insertion procedure may be associated with this finding. Perforation due to mucosal laceration or injury by the overtube around the ligament of Treitz during BAE has not been reported yet. However, the injury was severe, and exposure of the intestinal muscle layer was so obvious (Fig. 4). Even though perforation did not occur despite the severe mucosal injury, the mechanism or cases of colon perforation by the colonoscope shaft due to excessive loop formation during insertion should be considered. The operator should keep in mind that severe mucosal injury could occur owing to excessive loop formation during the insertion procedure.

\section{Decision on the insertion route}

The antegrade approach is recommended for lesions in the proximal two-thirds of the small bowel, while the retrograde approach is used for the distal one-third based on adjuvant information such as pre-enteroscopic capsule endoscopy, computed tomography enterography, and small bowel follow-through. ${ }^{12}$

\section{Sedation method}

Conscious sedation with midazolam and pethidine can be applied to antegrade or retrograde insertions such as esophagogastroduodenoscopy (EGD) and colonoscopy. However, because of the longer procedural time, maintenance of stable sedation state is not always possible. Patients often complain of recurrent belching, nausea, vomiting, and abdominal pain in antegrade insertion. In such cases, deep sedation with propofol is more appropriate and monitored anesthesia care by an anesthesiologist or general anesthesia can also be performed.

\section{Measurement of depth of insertion}

Several methods have been used to estimate the depth of insertion. The initial method was proposed by May et al. ${ }^{13}$ They estimated the insertion depth by using the Erlangen Endo-Trainer and validated the method during training courses. After each push-and-pull procedure, the operator recorded the length of the insertion. At the end of the procedure, all the recorded numbers were added. Through this method, they calculated the net advancement of the enteroscope. However, it has drawbacks because during the insertion procedure, slippage of enteroscope may occur and the unwanted withdrawal length cannot be determined exactly. Li et al. ${ }^{14}$ developed a simpler method by measuring the overtube insertion depth with maximal straightening at the level of the ligament of Treitz at the beginning of the procedure and at the location of an intestinal pathological finding. They estimated that every $5 \mathrm{~cm}$ of overtube advancement was equivalent to $40 \mathrm{~cm}$ of small-bowel advancement. Another method was proposed by Efthymiou et al. ${ }^{15}$ They measured the depth of insertion by counting the circular fold during the withdrawal process. By using a 5-cm marker inserted through the working channel of the enteroscope, they counted the number of folds per $5 \mathrm{~cm}$ during $5-\mathrm{cm}$ withdrawal steps. Through this method, a simple equation to calculate insertion depth was proposed as follows: depth in centimeters $=0.9 \times$ number of folds counted during withdrawal. However, this method also has a drawback because during scope withdrawal, rapid backward slippage may occur. More studies are needed to validate our findings.

\section{Minimal requirement and learning curve}

No appropriate guidelines stating the minimum training required before learning enteroscopy have been established. However, the trainee should have extensive experiences in EGD and colonoscopy before learning deep enteroscopy. According to the second International Consensus Statement on DBE, training should be undertaken by an advanced trainee only, considering the various difficulties during insertion and withdrawal of the enteroscope. The trainee should also have enough experience in not only diagnostic but also therapeutic procedures. These include hemostasis procedures using the endoclip, argon plasma, coagulation forceps, and epinephrine injection; polypectomy procedure, which is necessary in the management of small bowel tumors such as Peutz-Jeghers 
syndrome; and endoscopic balloon dilatation for stricture lesion. Experience in the ERCP procedure would be helpful. Sometimes, performing ERCP and biliary stent or drainage catheter insertion in a surgically altered anatomy is needed. As to the learning curve, enteroscopy through the oral route has a relatively faster learning curve, while the anal route approach seems to be more technically challenging and difficult to improve. For antegrade insertion, at least 10 cases are necessary under expert supervision; for retrograde insertion, 30-35 cases are needed to achieve an appropriate insertion depth. ${ }^{16-18}$ However, only few publications on the learning curve of enteroscopy are available, and the skill of individual trainees may differ, so these numbers might vary.

\section{CONCLUSIONS}

BAE is an evolving area of gastrointestinal endoscopy, and indications are expanding from only the diagnostic procedure of obscure gastrointestinal bleeding, inflammatory bowel disease, and small bowel tumor to an advanced therapeutic procedure. As the indications are expanding, more enteroscopy procedures are needed. Enteroscopy is generally performed by two persons who must cooperate closely. However, considering the transition from two- to one-person methods in colonoscopy, an enteroscopy procedure by one person might be popular in the near future. As to fluoroscopic guidance, with the accumulation of experiences, the use of guidance also decreased. With regard to enteroscopic learning, only few guidelines and studies are currently present. However, to acquire the skills to perform enteroscopy safely and effectively, standardized guidelines stating the minimum training requirement and quality indicators are needed in the future.

\section{Conflicts of Interest}

The author has no financial conflicts of interest.

\section{REFERENCES}

1. Hounnou G, Destrieux C, Desmé J, Bertrand P, Velut S. Anatomical study of the length of the human intestine. Surg Radiol Anat 2002;24:290-294
2. Hiratsuka H, Hasegawa M, Ushiromachi K, Endo T, Suzuki S, Nishikawa F. Endoscopic diagnosis in the small intestine. Stomach Intestine 1972;7:1679-1685.

3. Tada M, Akasaka Y, Misaki F, Kwaie K. Clinical evaluation of a sondetype small intestinal fiberscope. Endoscopy 1977;9:33-38.

4. Pennazio M, Spada C, Eliakim R, et al. Small-bowel capsule endoscopy and device-assisted enteroscopy for diagnosis and treatment of small-bowel disorders: European Society of Gastrointestinal Endoscopy (ESGE) clinical guideline. Endoscopy 2015;47:352-376.

5. Yamamoto H, Sekine Y, Sato Y, et al. Total enteroscopy with a nonsurgical steerable double-balloon method. Gastrointest Endosc 2001;53:216220.

6. Tsujikawa T, Saitoh Y, Andoh A, et al. Novel single-balloon enteroscopy for diagnosis and treatment of the small intestine: preliminary experiences. Endoscopy 2008;40:11-15.

7. Matsushita M, Shimatani M, Okazaki K. Clockwise insertion: a risk factor of pancreatic hyperamylasemia and acute pancreatitis after peroral balloon-assisted enteroscopy. Dig Endosc 2016;28:482.

8. Araki A, Tsuchiya K, Okada E, et al. Single-operator double-balloon endoscopy (DBE) is as effective as dual-operator DBE. J Gastroenterol Hepatol 2009;24:770-775.

9. Kav T, Balaban Y, Bayraktar Y. The power suction maneuver in single-balloon enteroscopy. Endoscopy 2008;40:961-962; author reply 962.

10. Manno M, Mussetto A, Conigliaro R. Preliminary results of alternative "simultaneous" technique for single-balloon enteroscopy. Endoscopy 2008;40:538; author reply 539.

11. Despott EJ, Murino A, Fraser C. Management of deep looping when failing to progress at double-balloon enteroscopy. Endoscopy 2011;43 Suppl 2 UCTN:E275-E276.

12. Gay G, Delvaux M, Fassler I. Outcome of capsule endoscopy in determining indication and route for push-and-pull enteroscopy. Endoscopy 2006;38:49-58.

13. May A, Nachbar L, Schneider M, Neumann M, Ell C. Push-and-pull enteroscopy using the double-balloon technique: method of assessing depth of insertion and training of the enteroscopy technique using the Erlangen Endo-Trainer. Endoscopy 2005;37:66-70.

14. Li XB, Dai J, Chen HM, et al. A novel modality for the estimation of the enteroscope insertion depth during double-balloon enteroscopy. Gastrointest Endosc 2010;72:999-1005.

15. Efthymiou M, Desmond PV, Brown G, et al. SINGLE-01: a randomized, controlled trial comparing the efficacy and depth of insertion of singleand double-balloon enteroscopy by using a novel method to determine insertion depth. Gastrointest Endosc 2012;76:972-980.

16. Mehdizadeh S, Ross A, Gerson L, et al. What is the learning curve associated with double-balloon enteroscopy? Technical details and early experience in 6 U.S. tertiary care centers. Gastrointest Endosc 2006;64:740750.

17. Mehdizadeh S, Han NJ, Cheng DW, Chen GC, Lo SK. Success rate of retrograde double-balloon enteroscopy. Gastrointest Endosc 2007;65:633-639.

18. Tee HP, How SH, Kaffes AJ. Learning curve for double-balloon enteroscopy: findings from an analysis of 282 procedures. World J Gastrointest Endosc 2012;4:368-372. 